\title{
Susceptibility of the deviants
}

\begin{abstract}
Genetic variability in the form of micro evolutionary changes such as point mutations or macro evolutionary changes such as changes in the large segment of DNA of the microorganisms could ultimately affect the antimicrobial susceptibility of the organism. Not only acquired antibiotic resistance but intrinsic resistance is also not an exception to the evolution of the organisms for the survival which has been approved and documented by number of the clinical trials and minimum inhibitory concentration (MIC) studies. To prove and review this, in house study about mutants or altered phenotypes recovered from exudates, urine and blood with the uncommon susceptibility pattern was undertaken in my hospital. Final results were informed to the clinicians so as to check the compliance of the majority of the patients to un-recommended antibiotics due to known intrinsic resistance. The present study did help in the management of the patients though the testing of the panel of antibiotics is not advised by CLSI guidelines.
\end{abstract}

Keywords: genetic variability, intrinsic resistance, mutants (deviants), uncommon susceptibility pattern, management
Volume 7 Issue 6 - 2019

\section{Grishma Kulkarni \\ Lab Director and Microbiology Consultant, Medicover Hospitals, India}

\section{Correspondence: Grishma Kulkarni, M.D. Medical} microbiology, Lab Director and Microbiology Consultant, Medicover Hospitals, Sarovar Complex, 5-9-22, Secretariat Road, Saifabad, Khairtabad, Secunderabad, Telangana 500063, India,Tel 9I-040-8I06586972, Email drgrishma@gmail.com

Received: October 22, 2019 | Published: November 29, 2019

\section{Introduction}

Genetic variability is essential to occur evolution. The fitness of the organism depends on its capacity to adapt to the changing environmental conditions. Antimicrobial agents exert strong selective pressure on the bacterial population favoring the microorganisms that are capable of resisting them. Genetic variability may occur by variety of the mechanisms such as micro evolutionary changes in the form of point mutations and macro evolutionary changes in the large segment of the DNA which ultimately leads to acquired resistance and better survival of the organisms. ${ }^{1}$ Intrinsic resistance is also not an exception to this transformation as documented by HHS studies about Burkholderia pseudomallei and other Minimum inhibitory concentration (MIC) studies as well. Though B.pseuodomallei is known for its intrinsic resistance to aminoglycosides and macrolide, found to be susceptible to them due absence of or nonfunctional RND (resistance nodulation division) pumps.

\section{Materials and methods}

In the prospective study, total 1334 exudates, 1403 urine and 679 blood were received for culture and sensitivity during $1^{\text {st }}$ January 2018 to 31 December 2018 conducted at Medicover Hospitals, Secretariat, Hyderabad, India. All exudates and blood samples were inoculated on Blood agar, MacConkey agar and Urichrome agar and urine specimens on Cysteine Electrolyte Deficient Agar (CLED) and Urichrome agar and incubated for 48 hours under aerobic condition at $37^{\circ} \mathrm{C}$. All positive culture isolates were identified by conventional biochemical tests up to species level. Susceptibility was done by KB disc diffusion method as per CLSI guidelines. ${ }^{2,3}$ Antibiotics not be tested against respective organism because of known intrinsic resistance were also included in the study. Few isolates with common colony morphology and Gram stain appearance and the uncommon susceptibility pattern were subjected to automated organism identification system Vitek 2 for accurate identification and the unusual susceptibility patterns of the common organisms were informed to the clinicians as the final results.

\section{Results}

Only $41.82 \%$ of exudates, $46.18 \%$ of urine and $16.78 \%$ of blood cultures were positive and all these cultures did support the growth of total $23.40 \%$ of gram-positive cocci, $79.69 \%$ of gramnegative bacilli, $0.45 \%$ of Gram-positive bacilli and $9.54 \%$ of fungal pathogens. Out of $41.82 \%$ positive exudates cultures, majority of the mutants were Gram negative bacilli. Amongst them, Pseudomonas aeruginosa was frequently isolated, followed by Enterococcus fecalis, Acinetobacter.baumannii, Enterococcus faecium, Morganella morganii and others as depicted in Table 1. Most of the mutants isolated from positive urine cultures were Gram negative bacilli such as P.aeruginosa (1.38\%) followed by gram positive cocci such as E.fecalis $(1.08 \%)$ and E.faecium $(0.92 \%)$ as displayed in the Table 2. In addition to the exudates and urine, mutants were also recovered from blood specimens as shown in Table 3.

\section{Discussion}

Antibiotics are precious commodity and should be preserved to treat the serious and life-threatening infections. Dosing regimens should be chosen by considering the in vitro antibiograms and evidencebased practice guidelines for clinical efficacy and to prevent the development of resistance. ${ }^{1}$ Susceptibility testing of aminoglycosides, cephalosporins and tigecycline etc.is contraindicated for Salmonella $s p$, enterococci and Pseudomonas $s p$ respectively due to intrinsic resistance as per the CLSI guidelines. In the present study, majority of mutant isolates or deviants were recovered from exudates followed by urine and blood in descending order. Though known for intrinsic resistance, these altered phenotypes showed uncommon susceptibility pattern. Similar in vitro and vivo correlating mutants, susceptibility results were seen in many studies. ${ }^{4-23}$

As per one school of thought, three RND pumps have been characterized in B. pseudomallei. AmrAB-OprA is expressed in most B.pseudomallei strains and responsible for intrinsic resistance to aminoglycosides and macrolides, and also confers some resistance 
to tetracyclines. Rare aminoglycoside susceptible B. pseudomallei environmental or clinical isolates either do not express AmrAB-OprB due to regulatory mutations, or lack the AmrAB-OprA operon as a result of a chromosomal deletion, or express a non-functional efflux pump due to mutations that affect the AmrB RND transporter. Several strains of $B$. pseudomallei lack AmrAB-OprA and are therefore aminoglycoside and macrolide susceptible as per Nierman et al. ${ }^{4}$

Mutation of B.pseudomallei gene (waaF) which encodes a protein required for LPS core oligosaccharide biosynthesis increases polymyxin B susceptibility which could be mistaken as in vitro false positive susceptibility otherwise unknown. ${ }^{4}$ Similarly, a blood isolate Burkholderia mallei was susceptible to all antibiotics tested during our study as depicted in table no 3. Precisely, mutations, deletion, additions, nonfunctional genes or loss of DNA fragments do cause altered intrinsic resistance pattern, could be responsible for varied susceptibility pattern seen in my prospective study. The majority of the resistance factors encoded by $B$. pseudomallei are also found in B. mallei, and Burkholderia thailandensis. However, B. mallei is generally more susceptible to antibiotics than B. pseudomallei, presumably because ongoing in-host evolution leads to genome reduction, including loss of antibiotic resistance determinants as per Nierman et al analysis. ${ }^{4}$

Two years long Brazilian study about the antibiotic resistance and molecular typing of the hospital acquired Pseudomonas aeruginosa sepsis cases from different NICU revealed that only $28 \%, 26 \%, 25$ $\%, 21 \%$ of seven distinct clones of Pseudomonas aeruginosa isolated from blood culture were resistant to cephalothin, chloramphenicol, cotrimoxazole and tetracycline respectively. Serotype E(O11) being be predominant followed by B,D and I. ${ }^{5}$ Not recommended by CLSI guidelines, colistin was tested against Burkholderia cepacia for adult and children with different concentrations by Minimum inhibitory concentration(MIC) method in the Northern Ireland study and found that $20 \%$ of BCC genomovar III were susceptible to colistin in case of children.

Likewise, epochal experience of USA clinician who treated all health care associated Pseudomonal cystitis and CAUTI with intact renal functions with doxycycline almost for 35 years though it is not considered as a therapeutic option. Almost $75 \%$ of these urine culture positive cases of UTI due to Pseudomonas aeruginosa were resolved completely with doxycycline alone. He also stated that doxycycline would be preferred over tetracycline to treat UTI with renal insufficiency. ${ }^{7}$ Also Mukherji et al and Musher et al boasted that oral tetracyclines eliminated this infection in $85 \%$ of the patients with normal renal function tests. Interestingly, Musher et al added that use of doxycycline and minocycline in human study yielded uniform results to conventional tetracycline with slight lower rate of eradication. ${ }^{8,9}$

Truly, the isolated Pseudomonas aeruginosa from exudates and urine displayed susceptibility to tigecycline, co-trimoxazole, tetracycline, chloramphenicol, ampicillin/sulbactam and so on as presented in table no 1 and 2. Comparably, 18 identified strains of Pseudomonas aeruginosa from diabetic foot ulcers were tested against ampicillin, norfloxacin, tetracycline, and co-trimoxazole at Chennai, ${ }^{10}$ which revealed only $83.3 \%$ and $66.6 \%$ of Pseudomonas aeruginosa showed resistance to tetracycline and co-trimoxazole respectively. India has the largest number of diabetic individuals and appreciably poor economic conditions; the analysis of intrinsically resistant organisms showing susceptibility to inexpensive forbidden drugs in diabetic foot infections assumes significance and thus helps us to prescribe low priced drugs. ${ }^{10}$

A clinical study of efficacy of trimethoprim/ sulfadiazine and trimethoprim/sulfamethoxazole in the treatment of enterococcal UTIs conducted by Sitzen and Rugendorff mentioned that approximately $85 \%$ of the patients on the respective dosage were cured microbiologically inspite of being intrinsically resistant. ${ }^{11,12}$ Indistinguishably, Nielsen et al. analysis of effect of trimethoprim in combination with sulfamethoxazole on enterococcal UTI displayed microbiological cure in $60 \%$ of the patients at follow-up on day $14 .{ }^{13}$ In addition to this, Stratford and Dixson presented that $87.5 \%$ of patients cleared enterococcal UTIs due to trimethoprim and sulfamethoxazole therapy on follow up. ${ }^{14}$ In similar fashion, the present paper enterococcal isolates did show in vitro susceptibility to cotrimoxazole, azithromycin, cefoperazone/sulbactam and meropenem etc. Out of that, two patients with significant bacteria because of E.faecium responded well to meropenem. Till date, few percentages of enterococci do respond to meropenem and cefoperazone/sulbactum clinically in our hospital.

The aminoglycosides are not recommended for treating enteric fever due to infection with Salmonella. enterica serovar Typhi. Based on the in vitro results of amikacin and gentamicin disc diffusion and agar dilution methods and time kill assays, Kolkata based study from 1991 to 2003 done on a total of 464 blood culture isolates of Salmonella enterica serovar Typhi isolates found to be effective against MDR $S$. enterica serovar Typhi infection clinically. ${ }^{15}$ Identical clinical studies done by Anand et al. showcased that the $60 \%$ epidemic strains of MDR S. enterica serovar Typhi responded to gentamicin therapy. Clinical cure of ciprofloxacin-resistant enteric fever with gentamicin, gentamicin and amikacin had been reported by Adhikari et al and Daga et al as well respectively. ${ }^{16-18}$ Therefore, our results emphasis on revising in vitro antimicrobial susceptibility testing guidelines to test the unreconcilable antibiotics based on clinical studies. Besides organisms, biofilm growth of Pseudomonas aeruginosa was inhibited after 3 hours treatment with 2x and 4x MICs and after 24 $\mathrm{h}$ treatment with tetracycline-loaded chitosan microspheres prepared by coacervation method than that prepared by water in oil emulsion method. ${ }^{19}$

Apart from this, 1993 Delhi based study about the changing pattern of multi drug resistance in typhoid fever stated that out of 76 patients, 12,51 and 9 patients responded to a combination of chloramphenicol and gentamicin, ciprofloxacin and combination of cefotaxime and amikacin respectively. ${ }^{18,20}$ Indian based Ludhiana study, a combination of cephloridine and gentamicin showed poor response $(43.48 \%)$ despite in vitro sensitivity for typhoid, in contrast to $100 \%$ response was observed by Koul et al. ${ }^{21,22}$ Besides this, ermB gene contributing to macrolide resistance detected by PCR was present only in $71.1 \%$ of Enterococcus faecium and $62.1 \%$ E. fecalis as per a Hospital-Based Study in China, ${ }^{23}$ could be the reason behind for isolation of few erythromycin and clindamycin susceptible strains in the present study. All such successful in vivo and in vitro correlating antimicrobial studies did support our prospective analysis and review of uncommon susceptibility pattern of the microorganisms.

\section{Conclusion}

The best hope for the future is the greater understanding of the evolution of the organism affecting the intrinsic as well as acquired resistance. Then it would be even easy to select the forbidden antibiotics 
depending on the invitro susceptibility pattern in the clinical practice though the respective organism is known to be intrinsically resistant. As per present study, the constellation of both intrinsic and acquired resistance mechanisms in the microorganisms combines to create a unique and often difficult challenge for the researchers and clinicians. Further study is necessary to understand the interplay of these factors and their effect on antimicrobial therapy and management of patients. This would help us in revising the antimicrobial testing guidelines.

\section{Acknowledgments}

None.

\section{Conflicts of interest}

Author declares that there is no conflict of interest.

\section{References}

1. Opal SM, Pop-Vicas A. Molecular mechanisms of antibiotic resistance in bacteria. In: Douglas M, Bennett's, Principles and Practices of infectious diseases. 8th ed. Elsevier Saunders: Canada; 2015. 235-251 $\mathrm{p}$

2. Collee JG, Marr W, Miles RS, et al. Culture of bacteria and tests for identification of bacteria. In: Mackie and McCartney practical medical microbiology. 14th ed. Churchill Livingstone: New York; 1996. 113$151 \mathrm{p}$.

3. Clinical and Laboratory Standards Institute (CLSI). Performance Standards for Antimicrobial Susceptibility Testing. 26th ed. Clinical and Laboratory Standards Institute: Wayne, PA; 2016.

4. Rhodes KA, Schweizer HP. Antibiotic Resistance in Burkholderia species. Drug Resist Updat. 2016;28:82-90.

5. Loureiro MM, de Moraes BA, Mendonca VL, et al. Pseudomonas aeruginosa: Study of Antibiotic Resistance and Molecular Typing in Hospital Infection Cases in a Neonatal Intensive Care Unit from Rio de Janeiro City, Brazil. Mem Inst Oswaldo Cruz. 2002;97(3):387-394.

6. Moore JE, Crowe M, Shaw A, et al. Antimicrobial resistance in B.cepacia at two regional cystic fibrosis centres in northern Ireland :Is there any need for synergy testing. J Antimicrob Chemother. 2001;48(2):319-321.

7. Cunha BA. Oral doxycycline for non-systemic urinary tract infections (UTIs) due to P. aeruginosa and other Gram negative uropathogens. Eur J Clin Microbiol Infect Dis. 2012;31(11):2865-2868.

8. Mukerji AC, Sharma MM, Taneja OP, et al. A clinical trial of alpha6-deoxyoxytetracycline (doxycycline)in the treatment of urinary tract infections. Chemotherapy. 1969;14(2):77-85.

9. Musher DM, Minuth JN, Thorsteinsson SB, et al. Effectiveness of achievable urinary concentrations of tetracyclines against "tetracyclineresistant” pathogenic bacteria. J Infect Dis. 1975;131 Suppl:S40-S44.
10. Sivanmaliappan TS, Sevanan M. Antimicrobial susceptibility patterns of Pseudomonas aeruginosa from diabetes patients with foot ulcers. Int $J$ Microbiol. 2011;2011:605195.

11. Wisell KT, Kahlmeter G, Giske CG. Trimethoprim and enterococci in urinary tract infections: new perspectives on an old issue. J Antimicrob Chemother. 2008;62(1):35-40.

12. Sitzen W, Rugendorff EW. Co-trimazine once daily in urinary tract infections in comparison with co-trimoxazole given twice daily-a double-blind randomized study. Infection. 1981;9(2):91-95.

13. Hamilton-Miller JM. Antibiotic treatment of enterococcal infection. Antimicrob Agents Chemother. 1989;33(6):989.

14. Stratford BC, Dixson S. Results of treatment of 108 patients using trimethoprim plus sulfamethoxazole. Med J Aust. 1971;1(10):526-531.

15. Mandal S, Mandal MD, Pal NK. In vitro activity of gentamicin and amikacin against Salmonella enterica serovar Typhi: a search for a treatment regimen for typhoid fever. East Mediterr Health $\mathrm{J}$. 2009;15(2):264-268.

16. Anand AC, Kataria VK, Singh W, et al. Epidemic multiresistant enteric fever in eastern India. Lancet. 1990;335(8685):352.

17. Adhikari PMR, Baliga S. Ciprofloxacin- resistant typhoid with incomplete response to cefotaxime. $J$ Assoc Physicians India. 2002;50:428-429.

18. Daga MK, Sarin K, Sarkar R. A study of culture positive multidrug resistant enteric fever-changing pattern and emerging resistance to ciprofloxacin. J Assoc Physicians India. 1994;42(8):599-600.

19. Mahmoud HA, Melake NA, El-Semary MT. Bactericidal Activity of Various Antibiotics versus Tetracycline-loaded Chitosan Microspheres against Pseudomonas aeruginosa Biofilms. Pharmaceut Anal Acta. 2012;S15:1-9.

20. Kalra SP, Naithani N, Mehta SR, et al. Current Trends in the Management of Typhoid Fever. Med J Armed Forces India. 2003;59(2):130-135.

21. Takkar VP, Kumar R, Khurana S, et al. Comparison of Ciprofloxacin versus Cephalexin and Gentamicin in the Treatment of Multi-drug Resistant Typhoid Fever. Indian Paediatr. 1994;31(2):200-201.

22. Koul PB, Murali PV, Sharma PP, et al. Multi- drug resistant Salmonella typhi infections Clinical profile and therapy. Indian Pediatr. 1991; 28(4):357-361.

23. Jia W, Li G, Wang W. Prevalence and Antimicrobial Resistance of Enterococcus Species: A Hospital-Based Study in China. Int J Environ Res Public Health. 2014;11(3): 3424-3442. 